\title{
Interstitial lung disease in scleroderma
}

\author{
RONALDO ADIB KAIRALLA
}

Pulmonary involvement is quite frequent in the development of progressive systemic sclerosis (PSS), a disease that affects the skin and internal organs and may present in various forms, especially as interstitial lung disease (ILD) or pulmonary hypertension (PH). ${ }^{(1)}$ The incidence of pulmonary involvement varies depending on the population and on the methodology used for the diagnosis, reaching up to $70 \%$ for $1 \mathrm{LD}$ and $50 \%$ for $\mathrm{PH}$ in autopsy studies. ${ }^{(2)}$ The presence of pulmonary disease is a determining factor for the quality of life, morbidity and mortality. ${ }^{(3-4)}$ In the most comprehensive study found in the literature, comprising more than 1000 patients, pulmonary involvement was responsible for $33 \%$ of deaths. ${ }^{(5)}$ In the past few years, this theme has been extensively studied from various aspects. However, a number of doubts persist. A prospective multicenter study is currently being conducted in the United States. ${ }^{(4)}$

Interstitial disease is more often accompanied by the diffuse cutaneous form of PSS, whereas PH is more common in the limited cutaneous form, but both diseases may occasionally be associated with both forms. Proper management of ILD accompanied by PSS is still uncertain. The fact that the disease is rare contributes to this problem since few centers have a relevant sample under long-term follow-up treatment. In addition, evolution is slow and the disease may remain stable for long periods. Furthermore, the presence of $\mathrm{PH}$ or involvement of other organs may cause confusion in the diagnosis. ${ }^{(1-3)}$ Factors that may signal a worse prognosis for ILD include presence of positive antitopoisomerase (anti-Scl-70) autoantibodies, diffuse cutaneous involvement, being AfricanAmerican, severe forms of the disease (revealed by computed tomography and pulmonary function tests) and rapid evolution to systemic disease $(<5$ years), as well as functional and radiological worsening during follow-up. ${ }^{(6)}$

A routine for the diagnostic evaluation of ILD should therefore be followed, especially in cases presenting the disseminated cutaneous form and
anti-Scl-70 positivity, in order to identify high-risk patients and thereby take appropriate therapeutic measures early. The principal technique used to identify ILD is high-resolution computed tomography (HRCT) scans, which presents higher sensitivity and specificity than do clinical, radiological and functional evaluations. ${ }^{(1-2)}$

Clinical evaluation must take into account the rate of progression of the disease since, in the majority of the studies, the most severe ILD cases appear within the first five years and, therefore, early pulmonary investigation must be encouraged. Dyspnea is present not only in ILD patients but also in other conditions such as $\mathrm{PH}$, arthritis, myopathy, restrictive thoracic cutaneous disease and poor general health. Dry cough, occasionally severe, is a symptom that is more closely related to the incidence of ILD, as are crackling rales. ${ }^{(1)}$ Intense interstitial alterations are easily identified on chest $\mathrm{X}$-rays. However, early alterations may pass unnoticed. ${ }^{(7)}$ Reductions in lung volume and lung diffusing capacity for carbon monoxide (DLCO) are the most frequent alterations identified during the functional evaluation. Nevertheless, they may be related to other PSS complications: volume reduction may be caused by thoracic cutaneous involvement or myopathy, whereas an isolated reduction in DLCO is usually related to $\mathrm{PH} .{ }^{(1-2)} \mathrm{In}$ terms of prognosis, functional alterations are significant markers of ILD progression during initial as well as sequential evaluations. . $^{(3,6)}$

The sensitivity of HRCT is higher than $90 \%$ for the detection of PSS-related ILD and is the main test for this diagnosis. The ground-glass pattern, which may be focal or diffuse, predominates, the reticular, honeycombing or traction bronchiectasis patterns being rarer. The correlation with histology shows the predominance of non-specific interstitial pneumonia (NSIP) with ground-glass pattern. The other patterns, including usual interstitial pneumonia (UIP), have been correlated with fibrotic alterations. ${ }^{(7)}$ This high sensitivity may cause confusion in the management of these patients and in the interpretation of studies 
since there is no simple, widely used method for the quantification of these tomographic alterations. Traction bronchiectasis, reticulated aspects and honeycombing aspects are considered indicators of poor prognosis. However, the main prognostic marker is the spread of the disease revealed in the HRCT scan that, combined with pulmonary function, better defines the evolution of the disease. ${ }^{(1)}$

The objective evaluation of inflammation/fibrosis can be achieved through bronchoalveolar lavage (BAL) or lung biopsy. Various studies have shown that increases in neutrophil and eosinophil numbers correlate with the extent of the disease seen on HRCT scans. However, the practical relevance of this information is limited and is restricted to study protocols. An aspect that merits investigation is eosinophilia in BAL as a marker of poor prognosis. ${ }^{(8)}$ Due to the new classification of idiopathic interstitial pneumonia, the predominant histological pattern for lung biopsy is the NSIP pattern and, very rarely, the UIP pattern. Although the presence of cellular NSIP is usually considered a marker of better prognosis, the practical relevance of this histological characterization has not been defined and, therefore, is not recommended as part of the routine. It should be restricted to occasional clinical and tomographic presentations and to cases in which infection or reaction to medication is suspected. ${ }^{(8)}$ Histological investigation is still an important tool for the investigation since a significant portion of cases cannot be classified. Histological aspects may contribute to a better understanding of the pathogenesis. Even the characterization of NSIP, with its various degrees of inflammation and fibrosis, still calls for further studies. ${ }^{(1-2)}$

Within the context of the great interest in this topic, Jezler et al. ${ }^{(9)}$ reported the initial pulmonary evaluation of 58 patients with PSS in this issue of the Jornal Brasileiro de Pneumologia (Brazilian Journal of Pulmonology). This is the largest Brazilian cohort study on this theme. Therefore, this study becomes a reference point for the management of our patients, especially if we take into consideration the ethnic variations observed in autoimmune diseases. The presence of ILD, defined through HRCT alterations, was observed in $30(51.7 \%)$ of the patients, which is a slightly smaller value than those reported in the literature, in a population with a mean time to diagnosis of PSS of 4.1 years, which reflects a definite incidence since ILD usually appears in the first five years of the disease.(3) The reduction of lung volumes and DLCO was observed in the functional evaluation. When it was compared to the group not affected by lLD, crackling rales and antiScl-70 positivity were significantly more prevalent in the ILD group. Although diffuse cutaneous disease was also more frequent, the difference was not significant. There was no difference in DLCO and baseline dyspnea index in the functional comparison. However, lung volumes were significantly lower in the ILD group. This fact suggests the presence of $\mathrm{PH}$ in the group not affected with 1LD, which would explain the presence of dyspnea and the reduction in DLCO with no interstitial alterations. ${ }^{(1-2)} \mathrm{ln}$ the multivariate analysis, the best marker for the presence of ILD was FVC < $80 \%$, perhaps because it is the parameter that suffers less alterations in $\mathrm{PH}$, thus differentiating the two groups quite well.

Excluding the slightly lower incidence, the results of this study were similar to those in the international literature, ${ }^{(1-2)}$ showing that the behavior of the interstitial lung lesion in the Brazilian population is similar to that seen in other populations. The most significant difference was related to the pattern of tomographic alteration. Most studies reported a strong predominance of the ground-glass pattern, ${ }^{(2,7)}$ whereas Jezler et al. found a higher frequency of bronchiolectasis and honeycombing, patterns which correlate with worse therapeutic response. ${ }^{(1)}$ Since no quantitative analysis was carried out, these scarring-related alterations may be focal, whereas the ground-glass pattern is usually more diffuse. ${ }^{(7)}$

Choosing the best moment to initiate treatment as well as deciding which medications should be used in the treatment of ILD accompanied by scleroderma, is still controversial. ${ }^{(1,3)}$ An interesting algorithm proposes that, in the presence of ILD on HRCT scans, the type of tomographic pattern and the intensity of the functional injury should be taken into account. Therefore, in the presence of predominant patchy ground-glass pattern, an FVC or DLCO $<75 \%$ would indicate the need for treatment, and if FVC or DLCO were $>75 \%$ or if the reticular pattern was predominant, treatment would be recommended only if there were functional worsening in the quarterly evolution. ${ }^{(2)}$ For any form of treatment, the extent of functional and tomographic alterations should be taken into consideration. ${ }^{(1)}$ 
Regarding therapeutic options, the present trend is to use a combination of a corticosteroid and an oral or intravenous cyclophosphamide. Although there has been no definitive study, there is reasonable evidence that this combination results in functional benefits, especially for those patients presenting patchy ground-glass pattern on HRCT scans..$^{(10-11)}$ However, the long-term effects of immunosuppressive therapy are still uncertain. A better understanding of the pathogenesis of the lung injury may lead to more specific therapeutic options. In light of this, some evidence suggests a correlation between esophageal lesion and ILD in scleroderma. ${ }^{(12)}$ Therefore, special attention must be given to measures designed to prevent bronchial aspiration.

\section{RONALDO ADIB KAIRALLA}

Ph.D. Professor of Pulmonology at the Faculdade de Medicina da Universidade de São Paulo (FMUSP, University of São Paulo School of Medicine), São Paulo (SP), Brazil

\section{REFERENCES}

1. Latsi Pl, Wells AU. Evaluation and management of alveolitis and interstitial lung disease in scleroderma. Curr Opin Rheumatol. 2003;15(6):748-55.

2. Sahhar J, Littlejohn G, Conron M. Fibrosing alveolitis in systemic sclerosis: the need for early screening and treatment. Intern Med J. 2004;34:626-38.

3. Steen VD, Medsger TA Jr. Severe organ involvement in systemic sclerosis with diffuse scleroderma. Arthritis Rheum. 2000;43(11):2437-44.

4. Khanna D, Clements PJ, Furst DE, Chon Y, Elashoff R, Roth
MD, et al. Correlation of the degree of dyspnea with healthrelated quality of life, functional abilities, and diffusing capacity for carbon monoxide in patients with systemic sclerosis and active alveolitis: results from the Scleroderma Lung Study. Arthritis Rheum. 2005;52(2):592-600.

5. Ferri G, Valentini G, Cozzi F, Sebastiani M, Michelassi C, La Montagna G, et al. Systemic sclerosis: demographic, clinical and serological features and survival in 1,012 Italian patients. Medicine (Baltimore). 2002;81(2):139-53.

6. Morgan C, Knight C, Lunt M, Black CM, Silman AJ. Predictors of end stage lung disease in a cohort of patients with scleroderma. Ann Rheum Dis. 2003;62(2):146-50.

7. Desai SR, Veeraraghavan S, Hansell DM, Nikolakopolou A, Goh NS, Nicholson AG, Colby TV, et al. CT features of lung disease in patients with systemic sclerosis: comparison with idiopathic pulmonary fibrosis and nonspecific interstitial pneumonia. Radiology. 2004;232(2):560-7.

8. Bouros D, Wells AU, Nicholson AG, Colby TV, Polychronopoulos V, Pantelidis P, et al. Histopathologic subsets of fibrosing alveolitis in patients with systemic sclerosis and their relationship to outcome. Am J Respir Crit Care Med. 2002;165(12):1581-6.

9. Jezler S, Santiago MB, Lessa T, Araujo Neto C, Braga H, Cruz AA. Comprometimento do interstício pulmonar em portadores de Esclerose Sistêmica Progressiva (ESP): estudo de uma série de 58 casos. J Bras Pneumol. 2005;31(4)300-6.

10. White B, Moore WC, Wigley FM, Xiao HO, Wise RA. Cyclophosphamide is associated with pulmonary function and survival benefit in patients with scleroderma and alveolitis. Arch Intern Med. 2000;132(12):947-54.

11. Pakas I, loannidis JP, Malagari K, Skopouli FN, Moutsopoulos HM, Vlachoyiannopoulos PG. Cyclophosphamide with low or high dose prednisolone for systemic sclerosis lung disease. J Rheumatol. 2002; 29(2):298-304.

12. Marie 1 , Dominique $S$, Levesque $H$, Ducrotte $P$, Denis $P$, Hellot MF, et al. Esophageal involvement and pulmonary manifestations in systemic sclerosis. Arthritis Rheum. $2001 ; 45(4): 346-54$. 